\title{
Optical Charging of Self-Assembled InAs/GaAs Quantum Dots
}

\author{
K. F. Karlsson ${ }^{1, *}$, E. S. Moskalenko ${ }^{1, * *}$, P. O. Holtz ${ }^{1}$, B. Monemar ${ }^{1}$, W. V. Schoenfeld ${ }^{2}$, J. M. Garcia ${ }^{2}$ and P. M. Petroff $^{2}$ \\ ${ }^{1}$ Department of Physics and Measurement Technology, Linköping University, S-581 83 Linköping, Sweden \\ ${ }^{2}$ Materials Department, University of California-Santa Barbara, Santa Barbara, California 93106, USA
}

Received June 17, 2001; accepted November 19, 2001

PACS Ref: 78.67.He, 71.35.-y, 78.55.Cr

\begin{abstract}
It is demonstrated that the photoluminescence spectra of single self-assembled quantum dots are very sensitive to the experimental conditions, such as excitation energy and crystal temperature. A qualitative explanation is given in terms of the effective diffusion of the photogenerated carriers, determined by the experimental conditions, which influence the capture probability and hence also the charge state of the quantum dots. This is proposed as an effective tool to populate single quantum dots with extra electrons, by purely optical means, in order to study phenomena involving charged excitons.
\end{abstract}

\section{Introduction}

Quantum dots (QDs) confine carriers in all three directions on the nanometer length scale. This implies a zero-dimensional density-of-states, similar to atoms, which is believed to improve the performance of electro-optical applications such a semiconductor lasers. In addition, the strong confinement results in an increased interaction between the trapped carriers, which also makes QDs interesting for single-electron devices. Consequently, multiparticle complexes in QDs are important to understand because they will influence the performance of QD-based devices.

Multiparticle complexes include multiexcitons and charged excitons. Charged excitons in QDs have recently been studied theoretically [1-3], and experimentally in large ensembles [3]. However, the inhomogeneous broadening present in measurements of QD ensembles, caused by the inevitable size distribution, conceals features corresponding to the charging effects on the $\sim$ meV level. Very recently, experimental studies of the optical properties of charged excitons in single QDs systems were published, e.g. [4,5].

The present paper is devoted to the creation of charged excitons in single InAs/GaAs QDs by purely optical means, relying on the different diffusivity of the photogenerated electrons and holes in the barrier.

\section{Samples and experimental setup}

The samples studied were grown by molecular beam epitaxy on semi-insulating GaAs (100) substrates. On top of a $100 \mathrm{~nm}$ $\mathrm{nm}$ GaAs layer self-assembled lens-shaped QDs were formed on a wetting layer (WL) from about 1.7 ML InAs deposition, and thereafter covered by $100 \mathrm{~nm}$ GaAa. A certain sample region of very low dot density was chosen for our study where the interdot spacing well exceeded the resolving power of the micro-PL-setup used.

\footnotetext{
*e-mail: freka@ifm.liu.se

**Permanent address: A. F. Ioffe Physical - Technical Institute, Russian Academy of Science, 194021, Polytechnicheskaya 26, St. Petersburg, Russia.
}

The QDs were studied by means of a conventional microPL setup. To excite the QDs, a cw-Ti-Sp laser tunable between 700 and $900 \mathrm{~nm}$ was used. The laser beam was focused on the sample surface to a spot of diameter $2 \mu \mathrm{m}$ by a microscope objective. The luminescence signal was collected by the same objective and dispersed by a monochromator combined with a $\mathrm{LN}_{2}$ cooled Si-CCD camera. The temperatures used in the experiments were ranging from 4 to $40 \mathrm{~K}$.

\section{Experimental results and discussion}

\subsection{Peak identification}

Several QDs have been studied, but most of them exhibit a similar behavior and are well-represented by the spectra of the QD shown in the present paper. A typical low-temperature PL spectrum of a single QD is shown in Fig. 1(a), taken at a moderate excitation power $\left(P_{\mathrm{ex}}=42 \mathrm{nW}\right)$ with an excitation energy $\left(\hbar \omega_{\text {photon }}\right)$ above the barrier band gap $\left(E_{\mathrm{g}}=1.519 \mathrm{eV}\right)$.

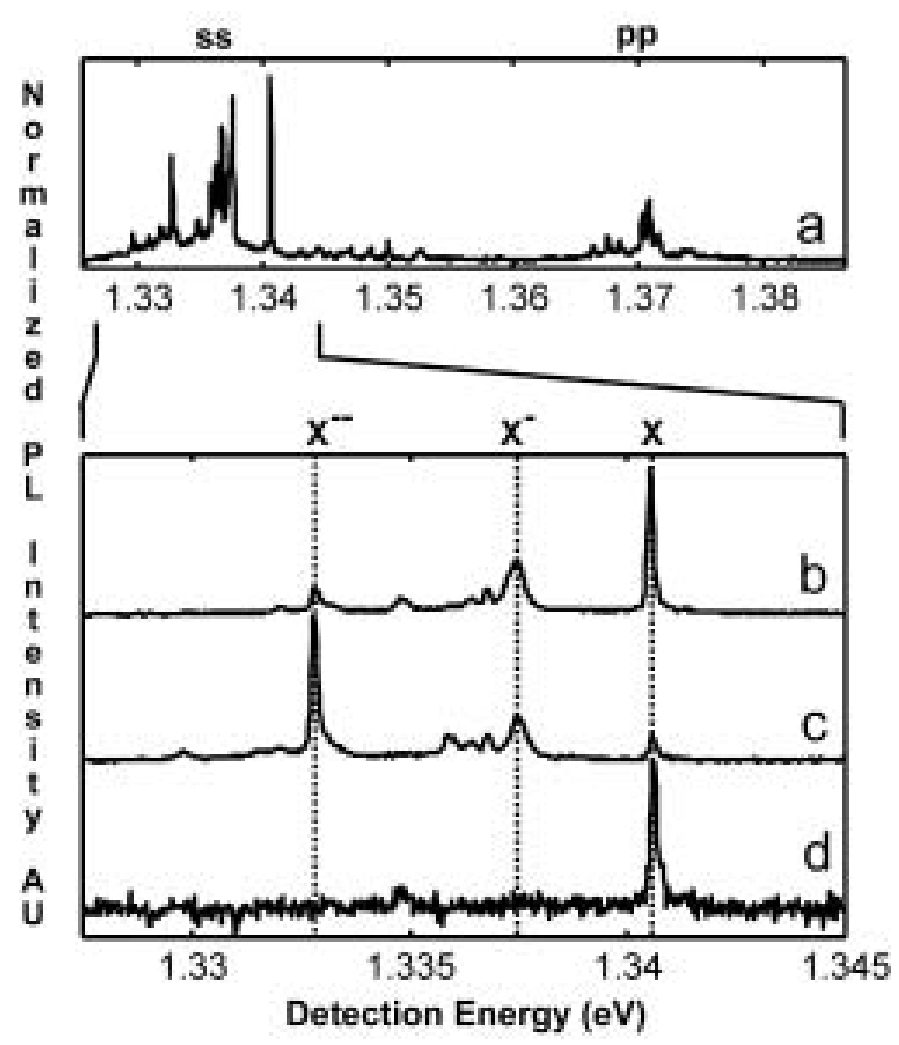

Fig. 1. Typical PL spectra of a single QD at a temperature of $4 \mathrm{~K}$ for different excitation energies above the barrier band gap, a) $\hbar \omega_{\text {photon }}=1.536 \mathrm{eV}$ $\left(P_{\mathrm{ex}}=42 \mathrm{nW}\right), \quad$ b) $\hbar \omega_{\text {photon }}=1.561 \mathrm{eV} \quad\left(P_{\mathrm{ex}}=10 \mathrm{nW}\right), \quad$ c) $\hbar \omega_{\text {photon }}=1.542 \mathrm{eV}$ $\left(P_{\mathrm{ex}}=10 \mathrm{nW}\right)$ and d) below the barrier and below the $\mathrm{WL}, \hbar \omega_{\text {photon }}=1.433 \mathrm{eV}$ $1.433 \mathrm{eV}\left(P_{\mathrm{ex}}=30000 \mathrm{nW}\right)$. 
The spectrum consists of two groups of emission lines, separated by $\sim 30 \mathrm{meV}$, denoted ss and pp, respectively. At low and moderate excitation powers $\left(P_{\mathrm{ex}}<100 \mathrm{nW}\right)$, the integrated intensity of the ss (pp) group exhibits linear (superlinear) dependence on the excitation power. At high power, the pp intensity exceeds the saturated ss intensity. On the basis of this characteristic power dependence, the group ss ( $\mathrm{pp}$ ) is attributed to transitions related to the ground states (first excited states) of the QD, i.e. transitions between electrons and holes, both in the $\mathrm{s}(\mathrm{p})$ shells.

The present paper is mainly focused on the ss transitions, which exhibit a dramatic dependence on the excitation energy, in the case of $\hbar \omega_{\text {photon }}>E_{\mathrm{g}}$. Figures 1(b) and 1(c) show two spectra of the ss emission of the same QD at two different excitation energies separated by $\sim 20 \mathrm{meV}$. Three peaks, denoted $\mathrm{X}, \mathrm{X}^{-}$and $\mathrm{X}^{-}$, are present in both spectra but with a remarkably different intensity distribution for the $\mathrm{X}$ and $\mathrm{X}^{-}$emissions. All three peaks are present at the lowest excitation power and grow linearly with the power, without essential intensity redistribution. Consequently, the peaks are not related to multiexciton complexes but to single-exciton occupation and correspoding charged complexes [6]. To distinguish the neutral exciton from the charged exciton, the sample was excited with an energy below the WL. The electrons and holes are in this case absorbed either into the QD directly or into localized states of the WL, whereby the population of the QD with an equal number of photogenerated electrons and holes is expected [6]. As shown in Fig. 1(d), only the $\mathrm{X}$ peak is present at excitation below the WL excitation, which therefore is ascribed the neutral exciton.

Calculations performed on lens-shaped QDs show that charged excitons, i.e. QDs with an extra electron (hole), in addition to the exciton, are red-shifted (blue-shifted) relative to the neutral exciton PL emission [7]. Since the peaks $\mathrm{X}^{-}$ and $\mathrm{X}^{-}$- are red-shifted with respect to $\mathrm{X}$ it is reasonable to interpret these peaks as negatively charged excitons, i.e. the QD is filled with extra electron(s). In order to estimate the binding energies of negatively charged excitons, a simple perturbation theory model developed in Ref. [3] was applied. The model assumes a parabolic lateral confining potential, which has been shown to mimic the potential of selfassembled QDs very well [1]. Using the characteristic potential parameters for electrons and holes $\left(\hbar \omega_{\mathrm{e}}\right.$ and $\left.\hbar \omega_{\mathrm{h}}\right)$ as input it is possible to obtain values of all interesting energies, i.e. (following the notation in [3]) the repulsion $E_{\mathrm{ss}}^{\mathrm{c}}$ (attraction $\left.E_{\mathrm{ss}}^{\mathrm{eh}}\right)$ between the s-electrons (the s-hole and the s-electron) and the exchange interaction $E_{\mathrm{sp}}^{\mathrm{x}}$ between the s-electron and the p-electron.

The potential parameters used in the calculation are estimated to be $\hbar \omega_{e}=\sim 20 \mathrm{meV}$ and $\hbar \omega_{\mathrm{h}}=\sim 10 \mathrm{meV}$, respectively, using the experimentally obtained energy difference of $\sim 30 \mathrm{meV}$ between the ss and pp transitions, [1,3]. Using the effective masses and the dielectric constant deduced for similar QDs in Ref. [3], the binding energy of one extra electron $E_{\mathrm{sp}}{ }^{\mathrm{eh}}-E_{\mathrm{ss}}{ }^{\mathrm{c}}$ is obtained to $2.6 \mathrm{meV}$ and the exchange energy is obtained to $E_{\mathrm{sp}}{ }^{\mathrm{x}}=4.9 \mathrm{meV}$. Comparing these values with the experimental values extracted from Fig. 1, $E_{\mathrm{X}}-E_{\mathrm{X}^{-}}=3.1 \mathrm{meV}$ and $E_{\mathrm{X}^{-}}-E_{\mathrm{X}^{--}}=4.7 \mathrm{meV}$, where $E_{P}$ corresponds to the spectral position of peak $P$, indicates that $\mathrm{X}^{-}\left(\mathrm{X}^{--}\right)$corresponds to the charged exciton, i.e. the single exiton together with one (two) extra electrons. It should be noted that this good agreement between the experimental and the theoretical values is reproduced for other QDs studied as well.

\subsection{Influence of carrier diffusion}

In order to further investigate the dependence of the QD spectrum on the excitation energy $\hbar \omega_{\text {photon, }}$, the PL spectrum was measured at different energies in the range from $1.52 \mathrm{eV}$ $\mathrm{eV}$ to $1.70 \mathrm{eV}$, i.e. above the barrier band gap. The intensities of $\mathrm{X}$ and $\mathrm{X}^{--}$exhibit a clear anticorrelated oscillating behavior with respect to $\hbar \omega_{\text {photon, }}$ as shown in Fig. 2 . A similar competition between a neutral and a charged exciton has been reported previously for excitation in the WL [8].

The dotted vertical lines in Fig. 2 correspond to the energies $E_{\mathrm{g}}+n \cdot \hbar \omega_{\mathrm{LO}}\left(1+m_{\mathrm{e}}{ }^{*} / m_{\mathrm{h}}{ }^{*}\right)$, where $n$ is an integer $(n=1,2,3$, or 4$), \hbar \omega_{\mathrm{LO}}=36 \mathrm{meV}$ is the GaAs LO-phonon energy and $m_{\mathrm{c}}{ }^{*}=0.067 m_{\mathrm{h}}{ }^{*}=0.45 m_{0}$ ) is the electron (heavyhole) effective mass in GaAs [8, 9]. Consequently, these energy values correspond to the excitation energies $\hbar \omega_{\text {photon }}$ at which the electron's part of the excess energy in GaAs is exactly an integer times $\hbar \omega_{\text {LO }}$, assuming parabolic band dispersion [10]. It should be noted that the lines coincide with the intensity maxima (minima) of the $\mathrm{X}\left(\mathrm{X}^{--}\right)$peak.

The oscillating dependence of the QD exciton intensity on the excitation energy $\hbar \omega_{\text {photon }}$ can be explained in terms of the well-known phonon relaxation processes of carriers [10]. The excess energy of the photo-generated carriers is mainly released either by fast [9] (few ps) emission of LO-phonons or by much slower emission of acoustic phonons. If the excess energy exactly fits with an integer number of $\hbar \omega_{\mathrm{LO}}$, the excess energy is very quickly released whereby the carrier becomes motionless at the band edge shortly after generation. On the other hand, if the excess energy does not fit with an integer number of $\hbar \omega_{\mathrm{LO}}$, it takes longer time to release the energy remaining after the LO-phonon emission. During this

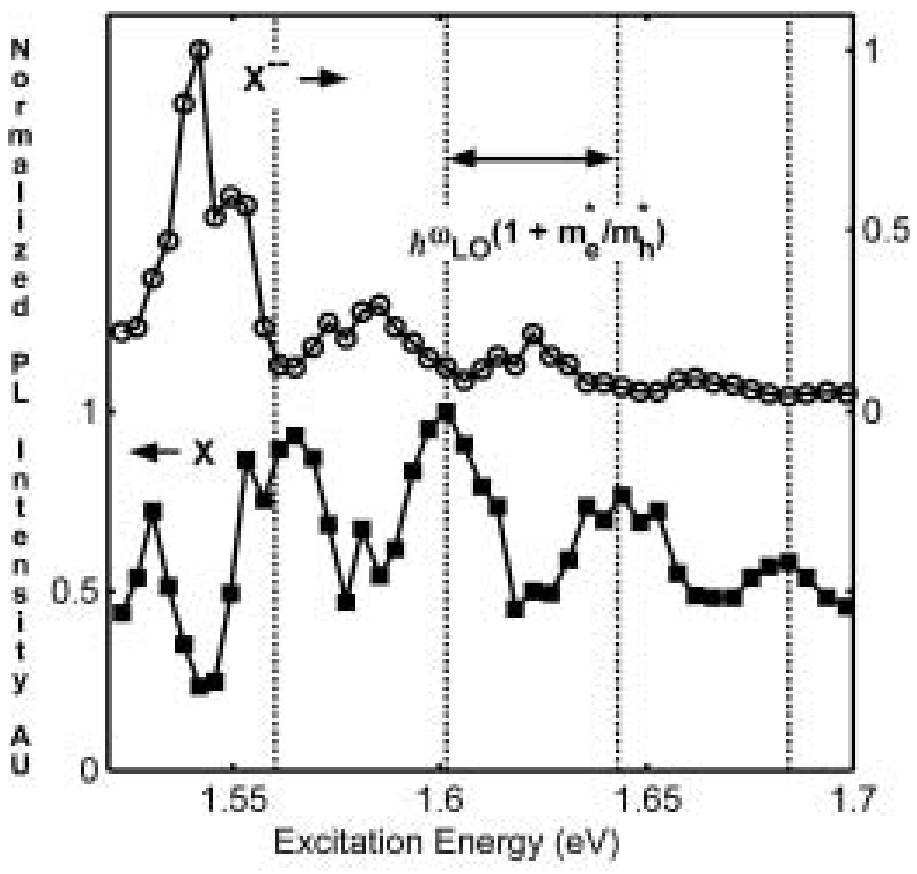

Fig. 2. The intensity dependence of the $\mathrm{X}$ and $\mathrm{X}^{--}$peaks in the QD ground state (ss) versus the above barrier band gap excitation energy, at a temperature of $4 \mathrm{~K}$. The dotted lines correspond to energies at which the excited electron's part of the excess energy is exactly an integer times the LO-phonon energy in the GaAs barrier. 
additional time the carrier is able move a longer distance in the crystal. In other words, excess energies that fit with $\hbar \omega_{\text {LO }}$ will result in the lowest effective diffusivity while energies that do not fit result in a higher diffusivity. Due to different masses, the excess energy is distributed in a non-equal way between the electrons and the heavy-holes, which in general also results in different diffusivities. Which type of carrier (electrons or holes) that obtains higher respectively lower diffusivity is, accordingly, determined by the excitation energy, and should exhibit an oscillatory behavior. Neglecting the Coulomb interaction between the carriers, the high diffusivity carriers move in a spatially larger region and are thus more likely to pass in the near vicinity of a strong capturing center (a quantum well or a QD) and become trapped. Consequently, the capturing center will initially primarily trap the high diffusivity carriers, but, since the Coulomb interaction cannot be neglected, the attraction of low diffusivity carriers of opposite charge will thereafter be increased. The steady-state condition at continuous excitation requires equal capture rate of electrons and holes. This condition is thus precisely fulfilled when the capturing center accommodates a certain number of extra carriers of higher diffusivity.

Another way to increase the effective diffusivity of the carriers is to increase the crystal temperature $T$ [11]. Since the electron mass is about seven times lighter than the heavy-hole mass in GaAs, the electrons gain higher diffusivity than the heavy-holes, when the temperature is increased. The PL spectra shown in Fig. 3 demonstrate the temperature dependence at three different excitation energies, $\hbar \omega_{\text {photon. }}$ Due to the small thermal shift of the GaAs band gap $(<0.5 \mathrm{meV})$, the excitation energy $\hbar \omega_{\text {photon }}$ was shifted by the same amount in the cases when $\hbar \omega_{\text {photon }}>E_{\mathrm{g}}$ to keep the excess energy imposed by the photoexcitation fixed. An analogous shift is also observed for the QD luminescence, as can be seen in Fig. 3. In Fig. 3(a), the excitation energy $\hbar \omega_{\text {photon }}$ is chosen to

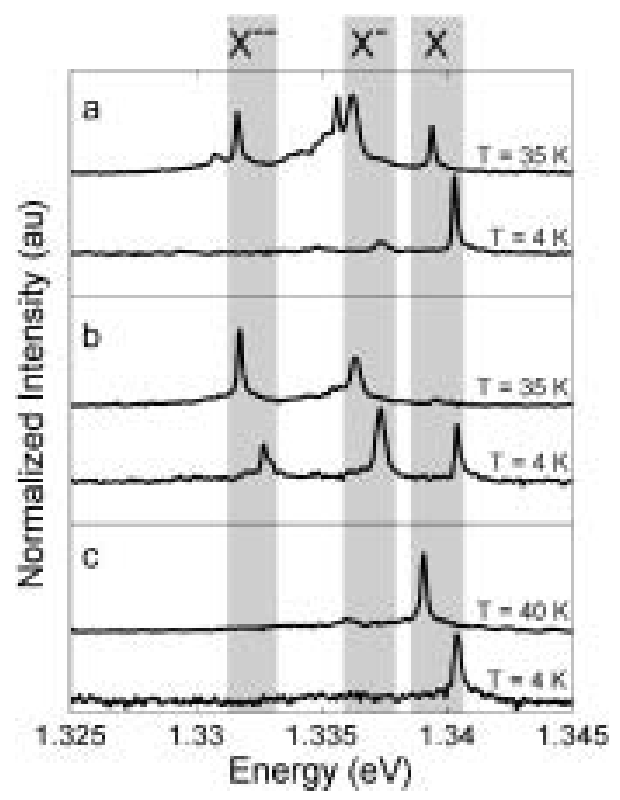

Fig. 3. The temperature influence on the QD ground state emission at excitation energies above the barrier band gap a) $\hbar \omega_{\text {photon }}=1.686 \mathrm{eV}\left(P_{\mathrm{ex}}=5 \mathrm{nW}\right)$ and b) $\hbar \omega_{\text {photon }}=1.557 \mathrm{eV}\left(P_{\mathrm{ex}}=5 \mathrm{nW}\right)$ and below the barrier and below the WL c) $\hbar \omega_{\text {photon }}=1.433 \mathrm{eV}\left(P_{\mathrm{ex}}=30000 \mathrm{nW}\right)$. give solely the pure exciton $\mathrm{X}$ at $4 \mathrm{~K}$. When the temperature is increased, there is a successive transfer of the intensity from $\mathrm{X}$, initially to $\mathrm{X}^{-}$, and subsequently also to $\mathrm{X}^{--}$. At $35 \mathrm{~K}$ the three peaks have comparable intensity. In Fig. 3(b), the excitation energy $\hbar \omega_{\text {photon }}$ is chosen to give comparable intensities for the three peaks at $4 \mathrm{~K}$. At $35 \mathrm{~K}$ the corresponding spectrum is dominated by $\mathrm{X}^{--}$, while $\mathrm{X}$ has vanished completely. This indicates that an increased temperature effectively fills the QD with extra electrons.

To finally confirm that the temperature effect is due to an increased diffusivity and not due to an activation of localized carriers the sample was excited with an excitation energy $\hbar \omega_{\text {photon }}$ below the WL emission. Since the photogenerated carriers in this case are localized and hence are totally immobile, no diffusion can take place. Accordingly no peak except the pure exciton $\mathrm{X}$ is expected in the spectrum even at higher temperatures. However, if the extra electrons are due to electron activation, the spectrum should qualitatively exhibit the same temperature behavior as for excitation energies $\hbar \omega_{\text {photon }}>E_{\mathrm{g}}$. Obviously, the spectra in Fig. 3(c) are temperature independent, (except for the small thermal redshift) which is in consistence with the explanation in terms of diffusivity.

\section{Conclusions}

Three peaks in the QD ground state have been identified as the neutral, single charged and double charged exciton, respectively, in good agreement with a simple theoretical model. It has been shown that the QD charge-state is very sensitive to the excitation energy and the crystal temperature. The sensitivity is qualitatively explained in term of the effective diffusivity of the photogenerated carriers, which affect the initial capture probability of the QD. This can be used as a new effective tool to create and study phenomena involving charged excitons.

\section{Acknowledgements}

One of the authors (E.S.M.) gratefully acknowledges financial support of the Swedish Institute within the Visby Programme and partial support of the Russian Academy of Sciences (Low-Dimensional Nanostructures 2001).

\section{References}

1. Wojs, A., Hawrylak, P., Fafard, S. and Jacak, L., Phys. Rev. B 54, 5604 (1996).

2. Hawrylak, P., Phys. Rev. B 60, 5597 (1999); Wojs, A. and Hawrylak, P., Phys. Rev. B 55, 13066 (1997).

3. Warburton, R. J. et al., Phys. Rev. B 58, 16221 (1998).

4. Hartmann, A., Ducommun, Y., Kapon, E., Hohenester, U. and Molinari, E., Phys. Rev. Lett. 84, 5648 (2000).

5. Findeis, F. et al., Phys. Rev. B 63, 121309 (2001).

6. Finley, J. J. et al., Phys. Rev. B 63, 073307-1 (2001).

7. Lelong, Ph. and Bastard, G., Solid. St. Commun. 98, 819 (1996).

8. Wolfe, J. P., Yoon, H. W., Wake, D. R. and Morkoc, H., Semicond. Sci. Technol. 7, B240 (1992).

9. Yoon, H. W., Wake, D. R. and Wolfe, J. P., Phys. Rev. B 54, 2763 (1996).

10. Shaw, R. W., Phys. Rev. B 50, 3283 (1971); Nakamura, A. and Weisbuch, C., Solid-State Electron. 21, 1331 (1978).

11. Yoon, H. W., Wake, D. R. and Wolfe, J. P., Phys. Rev. B 46, 13461 (1992); Hillmer, H. et al., Phys. Rev. B 39, 10901 (1989). 